\title{
Leveraging Trust Relationships in Digital Backchannel Communications
}

Syavash Nobarany

University of British Columbia

Vancouver, BC

nobarany@gmail.com

\section{Mona Haraty}

University of British Columbia

Vancouver, BC

haraty@gmail.com

\section{Sidney Fels}

University of British Columbia

Vancouver, BC

ssfels@ece.ubc.ca

Brian Fisher

Simon Fraser University

Surrey, BC

bfisher@sfu.ca

Copyright is held by the author/owner(s).

CHI 2011, May 7-12, 2011, Vancouver, BC, Canada.

ACM 978-1-4503-0268-5/11/05.

\begin{abstract}
Discussions during lecture can clarify lecture points for audience members and help them deepen their

understanding. However, the fast-pace of lectures and the large number of attendees can make these discussions impossible. Although digital backchannels have been used to address this problem, they present some drawbacks such as increasing distractions and not providing valuable information. We suggest incorporating audience members' levels of trust in the knowledge of other members into the design of backchannel communication systems. Based on this approach, we present methods and design considerations to overcome the aforementioned drawbacks of the previous backchannel communication systems.
\end{abstract}

\section{Keywords}

Backchannel, Trust-network, Social filtering, Classroom communication, Back channel.

\section{ACM Classification Keywords}

H.5.3 Group and Organization Interfaces: ComputerSupported Cooperative Work.

\section{General Terms}

Design, Human Factors 


\section{Introduction}

Discussions among audience members during a lecture is a method of cooperative learning that can increase breadth and depth of the learning experience and is considered as an active learning strategy. Audienceinitiated interactions in large lecture rooms can be impeded by various factors such as fast pace of lecture, weak communication skills, lack of self-confidence, and fear of asking unintelligent questions.

According to the constructivist learning theory, learning is an active process in which learners construct new ideas and clarify concepts based on their experiences and current knowledge through discussions by selecting and transforming information [10]. Computersupported backchannels are designed to facilitate audience discussions. They allow attendees to ask their questions and start discussions to clarify or complement the front-channel (instructor's presentation) without interrupting it. However, the negative effects of backchannels such as distracting audience and creating off-topic discussions are not negligible [4].

In this paper, we present two methods for incorporating audience members' trust in each others' knowledge (just trust from now on) into the design of systems supporting backchannel communications. We explain the implementation of a proof of concept prototype. Finally, we analyze the social and cognitive aspects of the two approaches to incorporating trust. The analysis is informed by results of a pilot study obtained through content analysis of backchannel discussions and followup interviews.

\section{Related Works}

Digital backchannels have been used in meetings [14], conferences $[6,8]$ and classrooms [15]. Backchan.nl is a web-based system designed for academic conferences that helps presenters to manage audience questions [6]. Backchan. $\mathrm{nl}$ is a hybrid of back and front channels, since it provides a ranked list of audience comments and questions based on the audience votes. A comprehensive study was conducted at UC Berkeley with a public chat environment in a graduate classroom to understand the effects of using backchannels in long term [15]. Their study reported both off-topic and constructive discussions in the backchannel communications. In another studyat UC San Diego, students used ActiveClass [11] as their classroom backchannel to anonymously ask questions, answer the polls, and give feedback to the instructor. Earlier efforts in this area are covered in [15] and a review on various backchannel functions and possible interactions is available in [3].

Previous studies have shown the negative effects of using backchannel communications on audience's attention [4,15]. However, backchannels may help audience to engage in the topic and not to lose track of the presentation $[11,13,15]$. All of the previous backchannel were in the form of public $[6,11,15]$ or private [14] chat rooms, each of which had different problems such as increasing students' cognitive load (mainly in public channels) and not providing helpful information to the information seekers (mainly in private channels).

\section{Leveraging trust in backchannels}

Trust-networks are social networks in which nodes represent users and directed edges represent trust 
relationships between users. Based on the existing relationships in a trust-network, the trust level for nonexistent relationships can be inferred using trust inference algorithms $[1,5,9]$. Trust-networks have been employed in several areas such as recommender systems [5], and collaborative search [2]. The concept of trust maps to various constructs such as confidence, reputation, credibility and reliability in different domains. For example, in trust-based recommender systems, trust can be interpreted as confidence in taste. We present two methods for incorporating trust into backchannel communications. In the first method, trust in each other's knowledge is used for devising a routing mechanism for outgoing messages. The second method applies trust as a filter on incoming messages.

\section{Routing outgoing messages}

To meet our information needs in everyday situations, we usually start with using the most available resources such as the Internet and our friends, whom we trust in their knowledge relevant to our need. Trust inference algorithms can be used to extend the limited list of helpful friends and determine the receivers of questions in a backchannel. Trust, in this context, can be interpreted as a user's trust in others' knowledge and helpfulness. The group of first-level trustees can be augmented using a trust inference algorithm. We use the concept of "trustee" instead of friend, because the nature of discussions are supposedly about the lecture topics and people tend to ask from their more knowledgeable and helpful peers.

Augmenting a user's group of trustees and facilitating the communication with people at different levels of his trust-network increases the availability of information. One of the main goals of using backchannels is to
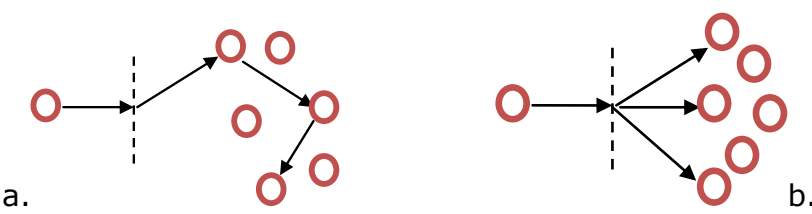

Figure 1. Trust-based message routing

$$
\begin{array}{ll}
\begin{array}{l}
\text { a. Incremental group } \\
\text { formation }
\end{array} & \text { b. Threshold-based group } \\
& \text { formation }
\end{array}
$$

decrease the intrinsic cognitive load of lecture materials caused either by their complexity or pace of the lecture; therefore, the accessibility of others' knowledge is a key factor in backchannels' effectiveness. Digital backchannels can be designed so that the users do not have to ask questions from a specific person; instead, they can send the question to their trust-network which will be routed through the network until someone can respond, or help the discussion (Fig. 1.a). This approach can substantially affect the audience discussions by providing opportunities for new and hard to initiate communications. The backchannel becomes a social catalyst that can be particularly valuable in conferences. However, the second approach (discussed later) can be more suitable when backchannel discussions are meant to be community discussions and the goal is to engage with a group of users.

We designed and developed a prototype of such backchannel to understand the possible implications of the design. In this prototype, a user can express an opinion or ask a question from his trust-network. Then, the message is routed to the first (most trusted) available trustee and if the receiver is unable to help (which can be determined by either party) the message 
will be passed to the next trustee and so on. The pilot study was conducted with 5 students in two 45-minute lectures, followed by interviews, to compare this design with a private backchannel (i.e. a private chat room).

\section{The Trust-enhanced Backchannel Prototype}

The goal of designing the proof-of-concept prototype was primarily enabling an exploratory study. The design decisions were made based on the basic functional and non-functional requirements, usability heuristics, and the available literature on designing digital backchannels. The prototype's interface consists of three tabs: trust management tab, history tab, and backchannel tab. In the trust management tab, students can express their trust to each other using a 5 -star rating widget and see their explicit and inferred trust relations (Fig. 2). Backchannel-tab shows the ongoing discussions (chat-boxes), and has a button for initiating a discussion. The backchannel discussions are not anonymous. Finally, the history tab allows reviewing the previous discussions. A chat-box has three possible states. Initial state is similar to a plain private chat-box. Asking state (Fig. 3.b) includes an "Ask someone else" button to be used when the current responder's answer is not sufficient to address the information need. Finally, answering state (Fig. 3.a.) includes "Pass it" button to be used by the responder when he cannot help the discussion. Both "Ask

someone else" and "Pass it" buttons cause the system

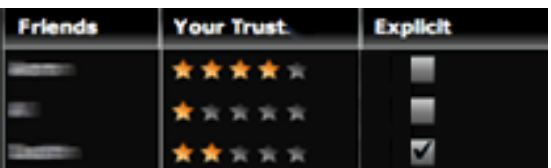

Figure 2. Trust management interface to search for the next trustee in the asker's trustnetwork.

\section{Communication multiplicity}

In this approach, outgoing messages are routed to the trustees who possibly may provide a helpful response. The number of trustees who receive the message and the level of their trustworthiness is an important factor that needs careful consideration. One option is to send the message to all of the people with a certain level of trustworthiness (Fig. 1.b) and initiate a group discussion on a topic. This method may lead to more discussions, but it may distract all of the participants in the discussion from the front channel. The alternative is to send the message to the peers, one by one based on their trustworthiness until the information need is satisfied (Fig. 1.a). The number of people involved in the discussion increases until the group solves the problem. This may impose the extraneous cognitive load on as few people as possible.

Transparency, Predictability and Controllability

Transparency and predictability of the trust inference algorithm was one of the issues we confronted in the pilot study. Three of the participants preferred to know who will respond them before asking a question and they wanted to know how exactly the trust inference algorithm is working. Another issue that arose during

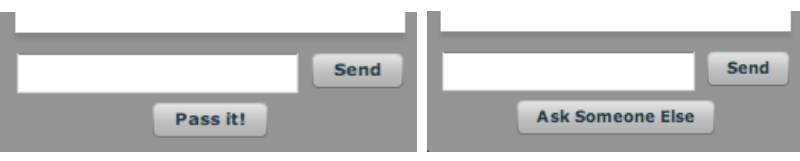

Figure 3. Different chat-box states

a. Responder's view

b. Asker's view 
the study was that students liked to keep each other responsible for answering their questions; because they were concerned about others using "Pass it" button when they hesitate to help. However, they liked to be able to use it as responders, when they are not sure about their response.

The design should deal with a trade-off between privacy and transparency, since unveiling the inference process requires exposing other users' relationships. One of the users mentioned that "knowing who is going to answer, informs the choices about what kind of language to use". Obtaining approval from the asker before sending the question to a new person could increase the controllability of the system. However, since we were in the exploratory phase of this research, we wanted to see users' reactions when receiving responses from people they are not friends with. Content analysis of the backchannel discussions showed that all of the users were able to satisfy their information needs regardless of who had the useful information, and one of the users mentioned that he liked "contacting someone you would not usually ask". Based on the interviews, the quality of answers in the trust-based backchannel were as good as in private backchannel and most of the users preferred to have the trustenhanced backchannel as an augmentation to instant messaging (private channel). A larger study is required to conclusively evaluate the effectiveness of this approach in reducing distractions and increasing the availability of information.

\section{Filtering incoming messages}

The second approach to leveraging trust relationships in backchannels is using them as information filter on incoming messages. Social relationships have been used in various information retrieval systems for filtering information $[7,12]$. Users need to be frugal in spending cognitive resources, mainly attention, on the backchannel communications not to lose track of the primary flow of information. Therefore, trust can play the role of an information filter to engage users only in the discussions that are worth paying attention.

One of the major usability issues of public backchannels is the relatively high frequency of messages, which may interrupt all of the users regardless of the usefulness of the information to them. A trust-based filter on the incoming messages can be used to deal with this issue. Users may decide to receive only messages from the users who are of a certain level of trustworthiness (as in Fig. 3), or in a more complex interface, the visual saliency of the backchannel messages can be adjusted based on the trustworthiness of their senders. In this approach trust to a person can be defined as the perceived probability of him sending useful or interesting messages to the backchannel.

This can be a way of reducing the number of interruptions and decreasing the extraneous cognitive load. Moreover, it helps preventing valuable messages going unnoticed, which is likely in public chat rooms due to the noise (i.e. non-useful messages). People may have different purposes for attending a lecture and messages that are less interesting for a specific attendee can be of importance to others. Therefore, determining the noise should be based on the personal trust-network rather than public measures such as reputation. The major drawback of this approach is that when someone hopes to get help from the more knowledgeable users, his message may go unnoticed, because they have not expressed their trust to him. 


\section{Conclusion}

The proposed mechanisms are just starting points for investigating the opportunities for designing sociallyaware digital backchannels. We designed a prototype of a trust-based backchannel, and conducted a pilot study to understand the implications of leveraging trust relationships in digital backchannels. By incorporating social relationships in design of digital backchannels, we can overcome some of the drawbacks of the public and private backchannels. The proposed approaches reduce the possibility of distraction, which was the major drawback of public backchannels. They also improve the accessibility of information in comparison with private backchannels. These enhancements can help resolving the trade-offs of using backchannels [6] in lecture-based learning environments.

\section{REFERENCES}

1. Audun, Roslan, and Colin. A survey of trust and reputation systems for online service provision. Decision Support Systems 43, 2 (2007), 618-644.

2. Briggs, P. and Smyth, B. Trusted search communities. Proceedings of the conference on Intelligent user interfaces, ACM (2007), 337-340.

3. Cogdill, S., Fanderclai, T., Kilborn, J., and Williams, M. Backchannel: whispering in digital conversation. Proceedings of the Hawaii International Conference on System Sciences, (2001), 8 pp.

4. Fried, C.B. In-class laptop use and its effects on student learning. Computers \& Education 50, 3 (2008), 906-914.

5. Golbeck, J.A. Computing and applying trust in webbased social networks. Doctoral dissertation. University of Maryland, College Park. 2005.

6. Harry, D., Green, J., and Donath, J. backchan.nl: integrating backchannels in physical space. Proceedings of the SIGCHI conference on Human factors in computing systems, ACM (2009), 13611370.
7. Massa, P. and Avesani, P. Trust-Aware Collaborative Filtering for Recommender Systems. In On the Move to Meaningful Internet Systems 2004: CoopIS, DOA, and ODBASE. 2004, 492-508.

8. McCarthy, J.F. and boyd, D.M. Digital backchannels in shared physical spaces: experiences at an academic conference. $\mathrm{CHI}$ '05 extended abstracts on Human factors in computing systems, ACM (2005), 1641-1644.

9. Nobarany, S., Haraty, M., and Cosley, D. GePuTTIS: General Purpose Transitive Trust Inference System for Social Networks. AAAI Spring Symposium on Social Information Processing, AAAI (2008), 66--71.

10. Palincsar, A.S. Social constructivist perspectives on teaching and learning. Annual review of psychology 49, 1 (1998), 345-375.

11. Ratto, M., Shapiro, R.B., Truong, T.M., and Griswold, W.G. The ActiveClass Project: Experiments in Encouraging Classroom Participation. Proceedings of Computer Support for Collaborative Learning, (2003).

12. Shardanand, U. and Maes, P. Social information filtering: algorithms for automating "word of mouth". Proceedings of the SIGCHI conference on Human factors in computing systems, ACM Press. (1995), 210-217.

13. Trimmel, M. and Bachmann, J. Cognitive, social, motivational and health aspects of students in laptop classrooms. Journal of Computer Assisted Learning 20, 2 (2004), 151-158.

14. Yankelovich, N., McGinn, J., Wessler, M., Kaplan, J., Provino, J., and Fox, H. Private communications in public meetings. $\mathrm{CHI}$ '05 extended abstracts on Human factors in computing systems, ACM (2005), 1873-1876.

15. Yardi, S. The role of the backchannel in collaborative learning environments. Proceedings of the international conference on Learning sciences, International Society of the Learning Sciences (2006), 852-858. 